\title{
HOMOCLINIC INTERSECTIONS AND INDECOMPOSABILITY
}

\author{
MARCY BARGE
}

(Communicated by George R. Sell)

\begin{abstract}
The closure of a one-dimensional unstable manifold of a hyperbolic fixed point of a diffeomorphism having homoclinic points is, under mild assumptions, shown to be an indecomposable continuum. As a result, dynamical systems possessing such behavior cannot be modeled using inverse limits based on any simple space.
\end{abstract}

I. Introduction. In [W] R. F. Williams demonstrated that every hyperbolic, one-dimensional, expanding attractor for a discrete dynamical system is topologically conjugate to the induced map on an inverse limit space based on a branched one-manifold. Examples [B] show that some nontrivial one-dimensinal nonhyperbolic attractors are also conjugate to inverse limit systems over branched onemanifolds.

In a complicated attractor (or invariant set) one expects to see periodic points possessing homoclinic orbits. If this is the case in a nondegenerate way, our Theorem provides the existence of a topologically indecomposable invariant set for the dynamical system. As a corollary we find that in certain commonly occurring situations (at certain parameter values in the Hénon map, for example) there are one-dimensional attractors (or invariant sets) that are not conjugate to any inverse limit system over a branched one-manifold.

II. Let $F: M \rightarrow M$ be a $C^{1}$ diffeomorphism of the $m$-manifold $M$ and let $p \in M$ be a hyperbolic fixed point of $F$ with stable manifold $W^{s}(p)$ and one-dimensional unstable manifold $W^{u}(p)$. Let $W^{u+}(p)$ be one of the branches of $W^{u}(p)$ and assume that $F\left(W^{u+}(p)\right)=W^{u+}(p)$ (otherwise, replace $F$ by $F^{2}$ ).

By Hartman's Theorem there is a homeomorphism

$$
\psi: B_{1} \rightarrow M, \quad B_{1}=\left\{\left(x_{1}, \ldots, x_{m}\right) \in \mathbf{R}^{m} \mid \sum_{i=1}^{m-1} x_{i}^{2} \leq 1,0 \leq x_{m} \leq 1\right\}
$$

such that

$$
\begin{gathered}
\psi\left(\left\{\left(x_{1}, \ldots, x_{m-1}, 0\right) \mid \sum_{i=1}^{m-1} x_{i}^{2} \leq 1\right\}\right)=W_{\mathrm{loc}}^{s}(p), \\
\psi\left(\left\{\left(0, \ldots, 0, x_{m}\right) \mid 0 \leq x_{m} \leq 1\right\}\right)=W_{\mathrm{loc}}^{u+}(p)
\end{gathered}
$$

(the local stable and unstable manifolds of $p$, respectively) and $A=\psi^{-1} \circ F \circ \psi$ is linear where defined.

Received by the editors February 21, 1986.

1980 Mathematics Subject Classification (1985 Revision). Primary 58F 13, 58F22, 55F20, 54H20.

Key words and phrases. Homoclinic point, inverse limit, indecomposable continuum. 
We consider the following conditions on $F$ :

(A1) $\operatorname{cl}\left(W^{u+}(p)\right)=$ closure of $w^{u+}(p)$ is compact.

(A2) There is an arc $\alpha$ in $\psi\left(B_{1}\right) \cap W^{u+}(p)$ such that $\alpha \cap W_{\text {loc }}^{s}(p) \neq \varnothing$ and $\alpha \nsubseteq W_{\mathrm{loc}}^{s}(p)$.

(A3) There is an essential $m-1$ sphere, $S^{m-1}$, in $W_{\text {loc }}^{s}(p)-\{p\}$ such that $S^{m-1} \cap \operatorname{cl}\left(W^{u+}(p)\right)=\varnothing$.

By a continuum we will mean a compact and connected metric space. A continuum is said to be indecomposable if it is not the union of two proper subcontinua. Equivalently, a continuum is indecomposable if every proper subcontinuum has empty interior.

THEOREM. Suppose that $F: M \rightarrow M$ is a $C^{1}$ diffeomorphism with hyperbolic fixed point $p$ as above that satisfies (A1)-(A3). Then $\operatorname{cl}\left(W^{u+}(p)\right)$ is an indecomposable continuum.

ProOF. Let $C=\left\{\left(x_{1}, \ldots, x_{m}\right) \mid\left(x_{1}, \ldots, x_{m-1}, 0\right) \in \psi^{-1}\left(S^{m-1}\right), 0 \leq x_{n} \leq \varepsilon\right\}$ where $\varepsilon>0$ is small enough so that $\psi(C) \cap \operatorname{cl}\left(W^{u+}(p)\right)=\varnothing$. For $n \geq 0$, let $C_{n}=A^{n}(C) \cap B_{1}=\psi^{-1}\left(\operatorname{comp}\left(F^{n}(\psi(C))\right)\right)$ where $\operatorname{comp}\left(F^{n}(\psi(C))\right)$ denotes the connected component of $F^{n}(\psi(C)) \cap \psi\left(B_{1}\right)$ containing $F^{n}\left(S^{m-1}\right)$. Note that $\psi\left(C_{n}\right) \cap \operatorname{cl}\left(W^{u+}(p)\right)=\varnothing$ for all $n$ since $\operatorname{cl}\left(W^{u+}(p)\right)$ is invariant under $F$. The hyperbolicity of $F$ at $p$ guarantees that, given any $r, 0<r \leq 1$, there is an $n=n(r)$ large enough so that $C_{n}$ separates $\left\{\left(0, \ldots, 0, x_{m}\right) \mid 0 \leq x_{m} \leq 1\right\}$ from $\left\{\left(x_{1}, \ldots, x_{m-1}, 0\right) \mid \sum_{i=1}^{m-1} x_{i}^{2}=r\right\}$ in $B_{1}$.

Let $G: \mathbf{R}^{+} \cap\{0\} \rightarrow W^{u+}(p)$ be a continuous, one-to-one, onto parameterization of $W^{u+}(p)$. We claim that $\operatorname{cl}(G([y, \infty)))=\operatorname{cl}\left(W^{u+}(p)\right)$ for any $y \in \mathbf{R}^{+}$. Indeed, let $\alpha=G([x, z])$ with $G(x) \in W_{\text {loc }}^{s}(p)$ be as in (A2) and, for $n \geq 0$, let $\alpha_{n}$ be the connected component of $F^{n}(\alpha) \cap \psi\left(B_{1}\right)$ containing $F^{n}(G(x))$. Hyperbolicity of $F$ at $p$ implies that $\lim \sup \alpha_{n}=W_{\text {loc }}^{u+}(p)$. For all sufficiently large $n, \alpha_{n} \subseteq G([y, \infty))$ so that $\operatorname{cl}(G([y, \infty))) \supseteq W_{\text {loc }}^{u}(p)$. Thus

$$
\begin{aligned}
\operatorname{cl}\left(W^{u+}(p)\right) & =\operatorname{cl}\left(C \bigcup_{n \geq 0} F^{n}\left(W_{\mathrm{loc}}^{u+}(p)\right)\right) \\
& \subseteq \operatorname{cl}\left(\bigcup_{n \geq 0} F^{n}(\operatorname{cl}(G([y, \infty))))\right) \\
& =\operatorname{cl}\left(\bigcup_{n \geq 0} \operatorname{cl}\left(F^{n}(G([y, \infty)))\right)\right) \\
& \subseteq \operatorname{cl}(G([y, \infty))) .
\end{aligned}
$$

Now suppose that $\operatorname{cl}\left(W^{u+}(p)\right)$ is decomposable. Let $H \subseteq \operatorname{cl}\left(W^{u+}(p)\right)$ be a proper subcontinuum such that interior $(H)$, relative to $\operatorname{cl}\left(W^{u+}(p)\right)$, is nonempty. Then $H \cap W^{u+}(p) \neq \varnothing$ and, since $H$ is proper, we have from the preceding paragraph that for no $y \geq 0$ is $G([y, \infty))$ contained in $H$. We also have (from the preceding paragraph) that interior $\left(G\left(\left[y_{1}, y_{z}\right]\right)\right)$, relative to $\operatorname{cl}\left(W^{u+}(p)\right)$, is empty for all $0 \leq$ $y_{1}<y_{2}<\infty$. Thus there are numbers $0 \leq y_{1}<y_{2}<y_{3}<y_{4}$ such that $G\left(y_{1}\right), G\left(y_{3}\right) \notin H$ and $G\left(y_{2}\right), G\left(y_{4}\right) \in H$. Now let $N$ be large enough so that 
$F^{-N}\left(G\left(y_{i}\right)\right) \in W_{\mathrm{loc}}^{u+}(p)$ for $i=1,2,3,4$ and let $0 \leq x_{m}^{1}<x_{m}^{2}<x_{m}^{3}<x_{m}^{4} \leq 1$ be such that $\psi\left(\left(0, \ldots, x_{m}^{i}\right)\right)=F^{-N}\left(G\left(y_{i}\right)\right)$ for $i=1,2,3,4$. Since $F^{-N}(H)$ is closed in $M$ and $F^{-N}\left(G\left(y_{1}\right)\right), F^{-N}\left(G\left(y_{3}\right)\right) \notin F^{-N}(H)$, there is an $r, 0<r \leq 1$, such that

$$
\psi\left(\left\{\left(x_{1}, \ldots, x_{m-1}, x_{m}^{i}\right) \mid \sum_{j=1}^{m-1} x_{j}^{2} \leq r\right\}\right) \cap F^{-N}(H)=\varnothing
$$

for $i=1$ and $i=3$.

Given $r$ as above, let $n=n(r)$ be as in the first paragraph of this proof. Let $D=\left\{\left(x_{1}, \ldots, x_{m}\right) \mid\left(x_{1}, \ldots, x_{m}\right) \in C_{n}\right.$ and $x_{m}^{1} \leq x_{m}^{2} \leq x_{m}^{3}$, or $\sum_{j=1}^{m-1} x_{j}^{2} \leq r$ and $x_{m}=x_{m}^{1}$, or $\sum_{j=1}^{m-1} x_{j}^{2} \leq r$ and $\left.x_{m}=x_{m}^{3}\right\}$.

Then $\psi(D) \cap F^{-N}(H)=\varnothing$ and $\psi(D)$ separates $f^{-N}\left(G\left(y_{2}\right)\right)$ from $F^{-N}\left(G\left(y_{4}\right)\right)$ in $M$. But then $F^{-N}(H)$, and hence $H$ itself, is not connected.

Thus, no proper subcontinuum of $\operatorname{cl}\left(W^{u+}(p)\right)$ has nonempty interior and $\operatorname{cl}\left(W^{u+}(p)\right)$ is indecomposable.

III. In this section we apply our theorem to obtain the corollary mentioned in the Introduction.

Given a continuum $X$ and a point $x \in X$, the composant determined by $x, C_{x}$, is the union of all proper subcontinua of $X$ that contain $x$. If $X$ is indecomposable, the composants of $X$ partition $X$, there are uncountably many distinct composants, and each is dense in $X$ (see [HY]).

By a topological branched one-manifold we will mean a compact, connected metric space that is locally homeomorphic to a one-point union of finitely many arcs (open, closed, or half-open, half-closed). Given a branched one-manifold $K$ with metric $d$ and a continuous map $f: K \rightarrow K$, the inverse limit space $(K, f)$ is the space $(K, f)=\left\{\mathbf{x}=\left(x_{0}, x_{1}, \ldots\right) \mid x_{n} \in K\right.$ and $f\left(x_{n+1}\right)=x_{n}$ for all $\left.n \geq 0\right\}$ with metric $\mathbf{d}$ given by

$$
\mathbf{d}(\mathbf{x}, \mathbf{y})=\sum_{n=0}^{\infty} \frac{d\left(x_{n}, y_{n}\right)}{2 n} .
$$

$(K, f)$ is a continuum and the induced map on $(K, f)$ is the homeomorphism (onto) $\hat{f}:(K, f) \rightarrow(K, f)$ given by $\hat{f}\left(\left(x_{0}, x_{1}, \ldots\right)\right)=\left(f\left(x_{0}\right), x_{0}, \ldots\right)$.

Maps $f: X \rightarrow X$ and $g: Y \rightarrow Y$ of the topological spaces $X$ and $Y$ are said to be topologically conjugate if there is a homeomorphism $h$ from $X$ onto $Y$ such that $h^{-1} \circ g \circ h=f$.

In the corollary, we have in mind the dynamical situation pictured in Figure 1.

COROLLARY. Let $F$ be as in the theorem and in addition assume that $\mathrm{cl}\left(W^{u+}(p)\right)$ $\cap W^{s}(p) \subseteq W^{w+}(p)$. Then for no continuous map $f$ of a branched one-manifold $K$ is $\left.F\right|_{\mathrm{cl}\left(W^{u+}(p)\right)}$ topologically conjugate to the induced map $\hat{f}$.

PrOOF. Suppose there is a branched one-manifold $K$, a continuous map $f: K \rightarrow$ $K$, and a homeomorphism $h$ from $\operatorname{cl}\left(W^{u+}(p)\right)$ onto $(K, f)$ such that $h^{-1} \circ f \circ h=$ $\left.F\right|_{\mathrm{cl}\left(W^{u+}(p)\right)}$. Without loss of generality, we may assume that $f$ is onto, otherwise replace $K$ by $L=\bigcap_{n \geq 0} f^{n}(L)\left(\hat{f}:(K, f) \rightarrow(K, f)\right.$ and $\left.\hat{f}\right|_{L}=\left(L,\left.f\right|_{L}\right)$ are topologically conjugate). 


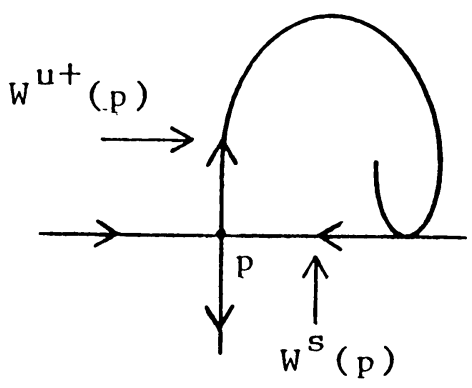

FIGURE 1

Since $W^{u+}(p)=\bigcup_{n>0} G([0, n])$ we see that the composant of $\operatorname{cl}\left(W^{u+}(p)\right)$ determined by $p$ in $\operatorname{cl}\left(W^{u+}(p)\right), C_{p}$, contains $W^{u+}(p)$. Thus, the additional assumption in this corollary means that the stable set of $p$ in $\operatorname{cl}\left(W^{u+}(p)\right)$, that is $\left\{x \in W^{u+}(p) \mid F^{n}(x) \rightarrow p\right.$ as $\left.n \rightarrow \infty\right\}$, is contained in $C_{p}$.

Thus, $S(h(p))=\{\mathbf{x} \in(K, f) \mid \hat{f}(\mathbf{x}) \rightarrow h(p)$ as $n \rightarrow \infty\}$ must be contained in $C_{h(p)}$, the composant determined by $h(p)$ in $(K, f)$.

Since $h$ conjugates $\hat{f}$ and $\left.F\right|_{c l\left(W^{u+}(p)\right)}$ and $p$ is a fixed point of $G, h(p)$ must be fixed by $\hat{f}$ so that $h(p)=\left(p_{0}, p_{0}, \ldots\right)$ for some $p_{0} \in K$.

It is clear from the definition of a branched one-manifold that there is an $l<\infty$ (depending of $K$ ) such that any collection of $l$ distinct points in $K$ separates $K$. Now let $\mathbf{x}=\left(x_{0}, x_{1}, \ldots\right) \in S(h(p))-\{h(p)\}$. Then $\mathbf{x}, \hat{f}(\mathbf{x}), \ldots, \hat{f}^{l-1}(\mathbf{x})$ are $l$ distinct points in $S(h(p))$. It follows that there is an $N \geq 0$ such that $\pi_{N}\left(\hat{f}^{i}(\mathbf{x})\right) \neq \pi\left(\hat{f}^{j}(\mathbf{x})\right)$ for $0 \leq i<j \leq l-1$ where $\pi_{N}$ is projection onto the $N$ th coordinate. Thus $K-\left\{\pi_{N}(\mathbf{x}), \ldots, \pi_{N}\left(f^{l-1}(\mathbf{x})\right)\right\}=K-\left\{x_{N}, f\left(x_{N}\right), \ldots, f^{l-1}\left(x_{N}\right)\right\}$ is not connected.

By the theorem, $(K, f)$ is indecomposable. Thus there is a composant $C$ in $(K, f)$ such that $C \cap C_{h(p)}=\varnothing$. In addition, $C$ is dense in $(K, f)$ and is connected. $\pi_{N}$ is continuous so that $\pi_{N}(C)$ is connected and dense in $K$. It must then be the case that $\pi_{N}(C) \cap\left\{x_{n}, \ldots, f^{l-1}\left(x_{N}\right)\right\} \neq \varnothing$. Say $\mathbf{y}=\left(y_{0}, y_{1}, \ldots\right) \in C$ and $i$, $0 \leq i \leq l-1$, are such that $\pi_{N}(\mathbf{y})-y_{N}=f^{i}\left(x_{N}\right)$.

Now, since $\mathbf{x} \in S(h(p)), \lim _{n \rightarrow \infty} f^{n}\left(x_{0}\right)=p_{0}$. Thus,

$$
\lim _{n \rightarrow \infty} f^{n}\left(y_{0}\right)=\lim _{n \rightarrow \infty} f^{n+N}\left(f^{i}\left(x_{N}\right)\right)=\lim _{n \rightarrow \infty} f^{n+i}\left(x_{0}\right)=p_{0}
$$

and it follows that $\mathbf{y} \in S(h(p))$. But this is impossible since $\mathbf{y} \in C, C \cap C_{h(p)}=\varnothing$ and $S(h(p)) \subseteq C_{h(p)}$. Thus, there can be no such $K, f$, and $h$.

\section{REFERENCES}

[B] M. Barge, A method for constructing attractors, preprint.

[HY] J. Hocking and G. Young, Topology, Addison-Wesley, 1961.

[W] R. F. Williams, One-dimensional non-wandering sets, Topology 6 (1967), 473-483.

Department of Mathematical Sciences, Montana State University, BozeMAN, MONTANA 59717-0001 